\title{
Exploration and Visualization of Computer Networks: Polyphemus and Hermes ${ }^{\star}$
}

\author{
Gabriele Barbagallo ${ }^{1}$, Andrea Carmignani ${ }^{1}$, Giuseppe Di Battista ${ }^{1}$, \\ Walter Didimo ${ }^{2}$, and Maurizio Pizzonia ${ }^{1}$ \\ 1 Dipartimento di Informatica e Automazione, Università di Roma Tre, Via della \\ Vasca Navale 79, 00146 Roma, Italy. \\ \{barbagal, carmigna, gdb, pizzonia\}@dia. uniroma3.it \\ 2 Dipartimento di Ingegneria Elettronica e dell'Informazione, Università di Perugia, \\ Via G. Duranti 93, 06125 Roma, Italy. didimo@diei.unipg.it
}

\section{Short Description}

Polyphemus and Hermes [1] are systems for exploring and visualizing computer networks at different abstraction levels. Today's Internet is organized as Autonomous Systems $(A S)$, each of them groups a set of networks under a single administrative authority (e.g. a private company). HERMES visualizes Internet as a set of ASes and interconnections between them. Polyphemus is a complementary system that is able to look inside an AS and to visualize its networks at the level of the routers and their physical links.

\section{Areas of Application}

The two systems presented in this paper are targeted to Internet service providers and to content delivery companies which need to have a clear view of the Internet structure in planning and designing their infrastructure.

\section{Layout Algorithms and Layout Features}

The drawing-server of POLYPhemus and the middle-tier server of HERMES both encapsulate a drawing engine that computes maps of the networks inside a single AS or draws parts of the ASes interconnection graph. The drawing engine is based on the GDToolkit library and has the following main features. Its basic drawing convention is the podevsnef model for orthogonal drawings having vertices of degree greater than four. However, since the graphs handled by HERMES often have many vertices (ASes) of degree one connected with the same vertex, the podevsnef model is enriched with new features for representing such vertices. The adopted algorithm implements a modified version of the standard topologyshape-metrics approach [1]. The user performs her exploration interactively and each step enriches the current map with new ASes and connections.

\footnotetext{
* Research supported in part by the Murst Project: "Algorithms for Large Data Sets". HERMES is available at http://www.dia.uniroma3.it/ hermes 


\section{Architecture and Interfaces}

For the two systems we have used different architectures. Since HeRmes uses publicly available data, it is targeted to offer a service to any Internet client. On the other hand, POLYPHEMUs requires to access private routing information. Hence, it is conceived as a local application that has to be installed and used by single users.

POLYPHEMUs is based on a two tiers architecture with a client and a drawingserver. The client (a Java application) allows the user to discover the network exploiting the knowledge of the routers that uses the Ospf routing protocol. Discovery is performed by accessing the Management Information Base (MIB) of the routers, with the Snmp protocol. Visualization of the the topology shows AS routers, networks grouped in Ospf areas, and inter-area relationships. For each item a rich set of information can be displayed.

HeRmes has a three tiers architecture. The user interacts with a top-tier client which forwards the user requests to a middle-tier server. The server translates the requests into queries to a repository (the bottom-tier). Using the toptier the user can interactively explore and visualize the ASes interconnections, and several information about ASes and their routing policies. HERMEs handles a large repository that is updated off-line from a plurality of sources [2]: APNIC, ARIN, RIPE, RADB, etc. The data in the repository are used by HERMEs to construct the ASes interconnection graph.

\section{Screenshots}

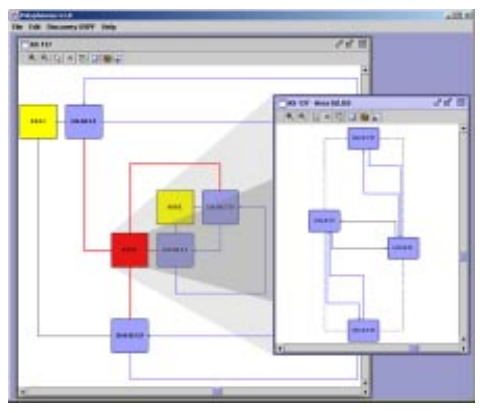

(a) Polyphemus

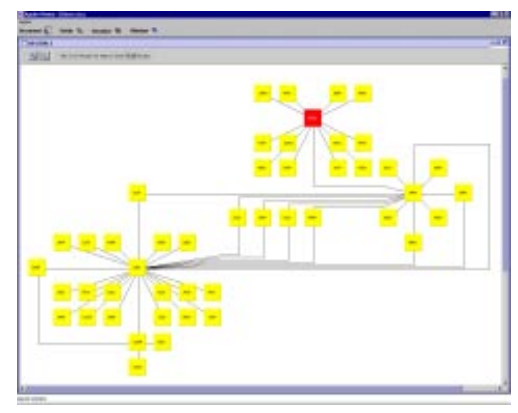

(b) Hermes

\section{References}

1. A. Carmignani, G. Di Battista, W. Didimo, F. Matera, and M. Pizzonia. Visualization of the autonomous systems interconnections with hermes. In J. Marks, editor, Graph Drawing (Proc. GD '00), volume 1984 of Lecture Notes Comput. Sci., pages 150-163. Springer-Verlag, 2000.

2. Merit Network, Inc. Radb database services. On line. http://www.radb.net. 